\title{
Atendimento remoto na APS no contexto da COVID-19: a experiência do Ambulatório da Comunidade da Escola Bahiana de Medicina e Saúde Pública em Salvador, Bahia
}

\author{
Andreia Beatriz Silva dos Santos, Marcus Vinicius Sacramento França, Juliane Lopes Ferreira dos \\ Santos
}

RESUMO

A COVID-19 é uma doença causada pelo SARS CoV-2, de variado espectro clínico, com alta transmissibilidade por via respiratória. No momento, a prevenção mostra-se essencial para enfrentamento dessa pandemia, assim como são necessários o distanciamento e regras de higiene. o novo coronavírus imprimiu uma realidade que demandou rápida adaptação do processo de trabalho dos profissionais nas unidades de saúde no que diz respeito aos cuidados primários em saúde. A telemedicina e suas modalidades vêm se destacando como meio de garantia da continuidade da assistência à saúde da comunidade, bem como para orientação e monitoramento de sintomáticos respiratórios, ratificando o papel da Atenção Primária e seus principais atributos, com destaque para o primeiro contato, a longitudinalidade e a integralidade. Este relato apresenta uma forma de organização da unidade docente-assistencial da Escola Bahiana de Medicina e Saúde Pública, em Salvador, Bahia, tendo o atendimento remoto como um dos instrumentos utilizados, revelando um novo caminho para a continuidade do cuidado da população, bem como um momento de aprendizado para os residentes do Programa de Residência em Medicina de Família e Comunidade, para a preceptoria e usuários do serviço durante a pandemia e que poderá ser incorporada no pós-pandemia por COVID - 19.

Palavras-chave: Atenção Primária à Saúde; Telemedicina; COVID-19; Medicina de Família e Comunidade; Assistência à Saúde.

\begin{abstract}
COVID-19 is a disease caused by SARS CoV-2, of varied clinical spectrum, with high respiratory transmissibility. At the moment, prevention is essential to face this pandemic, as well as distance and hygiene rules are necessary. The new coronavirus created a reality that demanded rapid adaptation of the work process of professionals in health units with regard to Primary Health Care. Telemedicine and its modalities have stood out as a means of guaranteeing the continuity of community health care, as well as for guidance and monitoring of respiratory symptoms, ratifying the role of Primary Care and its main attributes, with emphasis on the coordination of care and the longitudinality . This report presents a form of organization of a teaching-assistance unit of the Bahiana School of Medicine and Public Health, in Salvador, Bahia, with remote assistance as one of the instruments used, revealing a new path for the continuity of care for the population, as well as a learning moment for residents I doctors in Familiar Medicine, for the preceptorship and service users during the pandemic and which can be incorporated into the post-pandemic by COVID-19.
\end{abstract}

Keywords: Primary Health Care; Telemedicine; COVID-19; Familiar Medicine; Health Care.

Submissão recebida em 31 de maio de 2020.

Aceito para publicação em 08 de junho de 2020.

Avaliado pelo sistema Double Blind Review com participação dos editores.
Revista da Rede APS 2020

Publicada em: 09/06/2020

DOI:10.14295/aps.v2i2.120

Andreia Beatriz S. dos Santos (Escola Bahiana de Medicina e Saúde Pública, Salvador, BA, Brasil; Universidade Estadual de Feira de Santana, Feira de Santana, BA, Brasil)

Marcus Vinicius S. França (Escola Bahiana de Medicina e Saúde Pública, Salvador; BA, Brasil)

Juliane Lopes F. dos Santos (Escola Bahiana de Medicina e Saúde Pública, Salvador, BA, Brasil)

Correspondência para: Andreia Beatriz S. dos Santos andreiasantos72@hotmail.c om

Marcus Vinicius S. França marcus90franca@gmail.com Juliane Lopes F. dos Santos julianelfs@gmail.com 


\section{INTRODUÇÃO}

A COVID-19 é uma doença viral, causada pelo SARS-CoV-2, identificada no final de 2019, na província de Wuhan, China. O espectro clínico dessa morbidade varia desde casos leves $(80 \%$ dos casos) a casos graves, que evoluem com Síndrome Respiratória Aguda Grave, na minoria dos doentes. A transmissão viral ocorre principalmente por via respiratória, através de gotículas, esteja o paciente sintomático ou não (OMS, 2020). O período de incubação pode durar até 14 dias, mas geralmente acontece entre 5 e 6 dias (BRASIL, 2020).

As manifestações clínicas da COVID-19 caracterizam uma síndrome gripal - os pacientes podem apresentar congestão nasal, tosse, odinofagia, mialgia, fadiga, febre, dispneia, anosmia, ageusia, diarreia, entre outros sinais e sintomas. Dentre as complicações, cita-se a pneumonia elou síndrome respiratória aguda, choque e insuficiência renal (BRASIL, 2020).

No dia 30 de janeiro de 2020, a Organização Mundial de Saúde (OMS) declarou o surto por COVID-19 como uma emergência de Saúde Pública de importância internacional, tendo sido caracterizada, em 11 de março de 2020, como uma pandemia (OMS, 2020). Diante dessa situação excepcional foi forjada a necessidade de rápida adaptação dos diversos serviços para garantir a manutenção do cuidado à saúde das pessoas. Uma grande preocupação decorrente da pandemia pela COVID-19 deve-se a alta velocidade de contágio, levando ao risco de colapso os sistemas de saúde em todo o mundo, se desconsideradas medidas mitigadoras do contágio pelas sociedades atingidas, dentre as quais destacam-se o distanciamento social e regras de higiene.

Considerando-se o atual momento de pandemia pela COVID-19, a Atenção Primária à Saúde (APS) ganha maior relevância, necessitando que sejam reconhecidas as suas peculiaridades e necessidades para a prática do cuidado (BARBOSA \& SILVA, 2020). No Brasil, a forma como a APS está organizada vem possibilitando a realização da triagem dos pacientes suspeitos de síndrome gripal, a vigilância a saúde com notificação compulsória desses casos, a prescrição do isolamento domiciliar e monitoramento dos casos leves, assim como a estabilização e encaminhamento dos casos graves (BRASIL, 2020).

Por outro lado, a reorganização temporária da APS para o atendimento a demanda emergencial gerada com a pandemia pela COVID-19 e a adoção de medidas de mitigação do seu contágio, como a redução de consultas e procedimentos presenciais eletivos, têm gerado a necessidade da busca por ferramentas alternativas que permitam assegurar a manutenção do atendimento aos usuários que tenham outras condições e agravos à saúde que demandam cuidado continuado, ou seja, de maneira que não haja prejuízo para aqueles pacientes que necessitam de acompanhamento contínuo.

Nesse contexto, a telemedicina e a telessaúde apresentam-se como instrumentos estratégicos, considerando-se as recentes resoluções do Conselho Federal de Medicina (CFM, 2020) e do Conselho Federal de Enfermagem (COFEN, 2020), as quais são favoráveis ao uso dessas ferramentas em caráter excepcional e temporário, diante do atual cenário de emergência de saúde pública. Assim, o uso dessas tecnologias tem permitido o monitoramento dos pacientes em isolamento domiciliar com manifestações leves de COVID19, através de teleconsultas realizadas por profissionais da saúde. Mais recentemente, as teleconsultas médicas passaram a ser direcionadas também para o acompanhamento de paciente com doenças crônicas, visando minimizar a circulação não essencial de usuários nas unidades de saúde e, assim, reduzir os riscos de transmissão do vírus, permitindo ainda a identificação de sinais de gravidade ou de descompensação das doenças de base. (BRASIL, 2020). 


\section{SOBRE O LOCUS DE CUIDADO E PRÁTICA:}

\section{RELATO DA EXPERIÊNCIA}

O Ambulatório da Comunidade (AC) é uma unidade docente-assistencial da Escola Bahiana de Medicina e Saúde Pública (EBMSP), localizada no bairro de Brotas, na cidade de Salvador, Bahia., constituindo um locus de cuidado e campo de prática do Programa de Residência em Medicina de Família e Comunidade (PRMFC). Neste espaço são realizados os atendimentos dos usuários em nível de APS, com prioridade para a população residente nas áreas descobertas pela Estratégia de Saúde da Família (ESF).

No AC atuam quatro médicos residentes do primeiro ano do PRMFC, dois preceptores especialistas em Medicina de Família e Comunidade (MFC), além de equipe de enfermagem, profissionais da higienização e administrativos. A maioria dos atendimentos que ocorrem no AC são devido as doenças crônicas não transmissíveis, com destaque para Hipertensão Arterial Sistêmica e Diabetes Mellitus, ocorrendo também acompanhamento de gestantes e pacientes com transtornos mentais, sendo que crianças são atendidas em menor número. A partir dos encontros, das demandas e necessidades individuais e familiares das pessoas são instituídos os projetos terapêuticos.

O AC tem se estabelecido enquanto um equipamento de cuidado político-pedagógico com potencialidades para a prática de medicina de família e comunidade, do cuidado resolutivo e como possibilidade para a reorientação do modelo de atenção à saúde. Mesmo não sendo possível delimitar o território e de se integrar as Unidades de Saúde da Família (USF), este tem sido um importante locus para formação de Médicos de Família e Comunidade e para a consolidação de práticas de fortalecimento da APS.

Neste artigo, apresentamos um relato da experiência da reorganização docente- assistencial no Ambulatório da Comunidade da Escola Bahiana de Medicina e Saúde Pública, tendo a incorporação do teleatendimento como parte da estratégia de cuidado e como instrumento de atendimento remoto na APS, no contexto da COVID 19.

Após a confirmação do primeiro caso de coronavírus no estado da Bahia, no dia 06 de março de 2020, a coordenação do AC determinou a suspensão dos atendimentos presenciais em caráter temporário para a preservação da saúde da comunidade e dos diversos profissionais que atuam naquele espaço. Após a discussão dos detalhes para a reorganização do processo de trabalho com os atores envolvidos, levando em conta as necessidades de cuidados da população, procedeu-se o novo arranjo, através dos seguintes passos:

1.Revisão da lista de pacientes acompanhados no ambulatório da comunidade da Escola Bahiana de Medicina e Saúde Pública (EBMSP)

A seleção dos pacientes para atendimento ocorreu através da revisão dos prontuários eletrônicos dos pacientes acompanhados no AC, realizando-se uma análise retroativa de atendimentos ofertados. Foram avaliados a data da última consulta, morbidades, vulnerabilidades, demandas de exames e procedimentos em aberto e mais urgentes. Assim, foram definidos como critérios de prioridade para a realização das teleconsultas: pacientes com idade igual ou maior que 60 anos e presença de pelo menos uma doença crônica, aspectos que conferem maior risco de adoecimento da forma mais grave e morte por COVID 19. Posteriormente, foram selecionados também os pacientes com doenças crônicas de qualquer faixa etária.

\section{Contato com os pacientes prioritários para o atendimento}

O contato foi realizado para informar para os usuários o momento delicado da pandemia por COVID 19, as orientações das autoridades 
sanitárias de manter o distanciamento social, quanto ao uso de máscaras e as limitações existentes para o AC. Foram, então, informadas as possibilidades de atendimento remoto. Uma vez havendo concordância, agendou-se a consulta para o dia e horário compatível com a disponibilidade de horário de ambas as partes envolvidas. Não havendo concordância, foram agendadas consultas presenciais, reforçando-se os cuidados necessários sendo evitadas aglomerações no serviço de saúde.

\section{Realização do teleatendimento através de um} aplicativo

No dia e horário marcados, o aparelho celular da instituição foi devidamente disponibilizado para que preceptores e residentes realizassem a chamada através de um aplicativo de chamadas por vídeo. O prontuário médico foi revisado antes de cada chamada para atualizar a lista de problemas, identificar as pendências existentes e atualizar o projeto terapêutico de cada paciente. Foram informadas as limitações existentes, coletados os aspectos subjetivos, identificados resultados de exames a serem avaliados, realizada uma análise da situação, o surgimento ou não de novos problemas e finalizado atendimento com a construção coletiva de um plano terapêutico com as devidas pactuações.

4.Agendamento de pacientes com maiores necessidades de exame físico e demais orientações presenciais

Já durante as consultas foram identificados os pacientes que necessitariam de, pelo menos, um encontro presencial, tendo em vista limitações existentes para a realização do exame físico no atendimento a distância, a demanda de entrega de exames pendentes ou pelo fato do próprio paciente manifestar sua necessidade de se deslocar até o Ambulatório da Comunidade. Para pacientes idosos, foram discutidas estratégias que levaram em consideração o apoio de parentes, vizinhos ou outros membros da sua rede pessoal que pudessem se deslocar até o serviço para buscar receitas que necessitassem ser renovadas ou atualizadas ou ainda solicitações de exames.

\section{Perfil de atendimento via telemedicina dos pacientes no ambulatório da comunidade da EBMSP}

A análise do perfil dos pacientes foi feita com base na revisão dos prontuários dos pacientes atendidos entre os dias 23/04/2020 (início dos contatos para teleatendimento) e $28 / 05 / 2020$, totalizando 20 pacientes contactados e 23 consultas realizadas. Esses pacientes foram estratificados por idade, gênero, doenças e tipo de atendimento: presencial apenas, teleconsulta apenas ou teleconsulta seguida de presencial.

Dos 17 pacientes atendidos por teleconsulta, apenas 3 necessitaram de deslocamento até 0 ambulatório para realização de consulta presencial posterior, sendo todos para exame físico, a fim de elucidação diagnóstica ou modificação de prescrição medicamentosa de uso contínuo. Esse dado reforça a centralidade do exame físico na consulta médica para a tomada de decisões em casos de queixas novas, crônicas agudizadas e também no acompanhamento de alguns parâmetros a distância, a exemplo dos valores da pressão arterial, o que pode ser resolvido quando o paciente possui o equipamento para aferição a sua disposição.

Em relação aos atendimentos, houve predominância do atendimento de pacientes na faixa etária entre 61 e 70 anos e entre 41 e 50 anos, correspondendo a $35 \%$ dos atendimentos cada grupo. $85 \%$ dos pacientes atendidos por telemedicina corresponderam a mulheres. Nessa faixa etária, encontram-se as maiores prevalências de doenças crônicas, tais como hipertensão arterial, diabetes, dislipidemia e obesidade. Entre 51 - 60 anos, apenas 2 pacientes foram atendidos, sendo um por teleconsulta. Baseando-se em estatísticas de prevalência de doenças crônicas, esse grupo etário também deveria apresentar maior prevalência no atendimento. Uma limitação a 
ser considerada é o pouco tempo de uso do teleatendimento para a consulta. Entende-se que, com o passar do tempo, os números de pacientes atendidos nessa faixa etária se aproximem mais da realidade da população. A figura 1 indica a distribuição por faixa etária das teleconsultas.

Figura 1 - Distribuição de teleconsulta por faixa etária no Ambulatório da Comunidade

\begin{tabular}{|c|c|c|}
\hline \multicolumn{3}{|c|}{ Atendimentos no Ambulatório da Comunidade } \\
\hline Faixa etária & Teleconsulta & Presencial \\
\hline $0-10$ anos & 1 & 0 \\
\hline $11-20$ anos & 1 & 0 \\
\hline $21-30$ anos & 0 & 0 \\
\hline $31-40$ anos & 2 & 1 \\
\hline $41-50$ anos & 6 & 0 \\
\hline $51-60$ anos & 1 & 1 \\
\hline $61-70$ anos & 6 & 1 \\
\hline
\end{tabular}

Fonte: elaborado pelos autores (2020).

A análise do perfil de morbidades atendidas por telemedicina no AC revela o predomínio de pacientes hipertensos, obesos e dislipidêmicos, ou seja, parte das patologias elencadas como fator de risco para complicações por COVID-19 (figura 2). Outro aspecto importante é o fato de que os três pacientes que necessitaram de atendimento presencial após o teleatendimento possuíam doença crônica, demandando o exame físico para avaliar complicações da doença de base ou a necessidade de alteração da prescrição. Esse perfil de pacientes atendidos por telemedicina reforça a possibilidade de manter pacientes do grupo de risco em seus domicílios e garantir a manutenção do acompanhamento médico a fim de manter seu quadro clínico compensado. Segundo o Ministério da Saúde (2020), pacientes com doenças crônicas que não estejam compensadas constituem grupos de risco para complicações para SARS CoV-2.

Figura 2 - Percentual de pacientes atendidos via telemedicina por morbidades

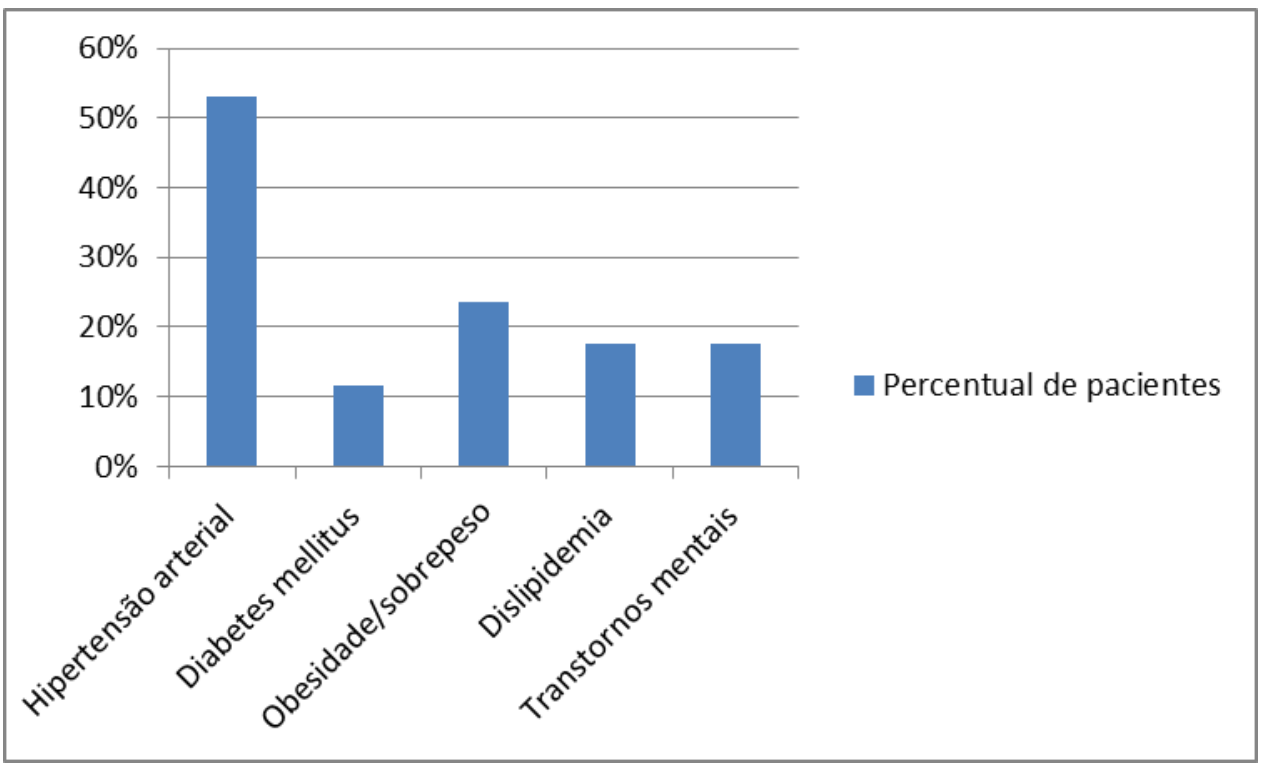

Fonte: elaborado pelos autores (2020) 


\section{CONSIDERAÇÕES FINAIS}

A pandemia por COVID-19 lançou novos desafios para o mundo, com destaque para os profissionais de saúde da Atenção Primária à Saúde. $O$ atendimento a distância pode se apresentar como uma alternativa diante das restrições impostas pelo isolamento social, distanciamento social e o cumprimento de quarentena, além de outras dificuldades que se impõem, a exemplo das limitações físicas e econômicas para o deslocamento até as unidades de saúde.

Os limites do atendimento à distância devem ser mais bem avaliados no sentido da construção de caminhos que viabilizem a sua prática. Entraves como a impossibilidade do exame físico, limitações de ordem técnica para o uso dos aplicativos e acesso as tecnologias digitais, nenhuma ou pouca privacidade durante as consultas a distância em residências com poucos cômodos, acústica limitada ou um número grande de moradores na mesma residência, devem ser avaliadas no sentido de não impactar a qualidade da consulta médica e das informações, assim como no sigilo médico.

O método empregado resguarda a possibilidade de manter o vínculo e o cuidado das pessoas neste momento delicado, no qual o deslocamento dos pacientes até a unidade e o compartilhamento de sala de espera por muitas pessoas representam um maior risco de transmissão da COVID-19, adoecimento e morte. Também pode contribuir para diminuir a sensação de abandono de algumas pessoas que, neste período, têm indicação de se manterem isolados de seus entes queridos e com as quais as equipes estão impossibilitadas de manter o contato físico previamente existente.

No contexto imposto pela pandemia por COVID19, a articulação ensino e serviço em uma unidade docente-assistencial tem possibilitado ampliar o campo de cuidado, saber e aprendizado para todos os envolvidos. A telemedicina impulsiona uma nova forma de pensar e construir o cuidado das pessoas na APS durante e no período pós-pandemia. A partir daí, serão necessárias discussões mais aprofundadas acerca das modalidades de telemedicina e detalhes sobre sua execução, buscando manter a qualidade das consultas, sigilo das informações dos pacientes e garantia de exame físico realizado por médicos, quando for preciso. 


\section{NOTAS E REFERÊNCIAS}

BARBOSA, S.; SILVA, A.V. A Prática da Atenção Primária à Saúde no Combate da COVID-19. APS em Revista. Belo Horizonte, v. 2, n. 1, p. 17-19, 2020.

BRASIL. Ministério da Saúde. Gabinete do ministro. Portaria n467, de 20 de Março de 2020. Dispõe, em caráter excepcional e temporário, sobre as ações de Telemedicina. Diário Oficial da União, Brasília, DF, 23 mar 2020.

BRASIL. Ministério da Saúde. Secretaria de Atenção Primária à Saúde. Protocolo de Manejo Clínico do Coronavírus na Atenção Primária a Saúde. Brasília, DF, mar 2020.

CATAPAN, S.C; CALVO, M.C.M. Teleconsulta: uma revisão integrativa da interação médico-paciente mediada pela tecnologia. Rev. bras. educ. med. Brasília, vol.44, n.1, 2020.

CONSELHO FEDERAL DE ENFERMAGEM. Resolução № 0634/2020, de 26 de março de 2020: Autoriza e normatiza, "ad referendum" do Plenário do Cofen, a teleconsulta de enfermagem como forma de combate à pandemia provocada pelo novo coronavírus (Sars-Cov-2), mediante consultas, esclarecimentos, encaminhamentos e orientações com uso de meios tecnológicos, e dá outras providências. Brasília: 2020. <Disponível em: http://www.cofen.gov.br/wpcontent/uploads/2020/03/RESOLU\%C3\%87\%C3\%830-COFEN-N\%C2\%B0-634-2020.pdf >. Acesso em 28 mai 2020.

CONSELHO FEDERAL DE MEDICINA. Ofício COJUR CFM no 1756/2020, de 19 de Março de 2020. Reconhece a possibilidade e a eticidade da utilização da telemedicina, além do disposto na Resolução CFM no 1.643, de 26 de agosto de 2002 <Disponível em: http://portal.cfm.org.br/images/PDF/2020_oficio_telemedicina.pdf>. Acesso em 28 maio 2020.

OMS. Organização Mundial de Saúde. Organização Pan-americana de Saúde. OMS declara emergência de saúde pública de importância internacional em relação a novo coronavirus. < Disponível em: https://www.paho.org/bra/index.php?option=com_content\&view=article\&id=6100:oms-declaraemergencia-de-saude-publica-de-importancia-internacional-em-relacao-a-novocoronavirus\&Itemid=812>. Acesso em: 26 mai 2020.

WEN CHAO LUNG. Telemedicina e Telessaúde: Uma abordagem sob a visão de estratégia de saúde apoiada por tecnologia. Atualidades Brasileiras em Telemedicina e Telesaúde, São Paulo, v. 2, p. 3-5, 2006. 